\title{
Unlocking the complexity of HIV and Mycobacterium tuberculosis coinfection
}

\author{
Eileen P. Scully' and Bryan D. Bryson ${ }^{2,3}$ \\ IJohns Hopkins University, Department of Medicine, Division of Infectious Diseases, Baltimore, Maryland, USA. ${ }^{2}$ Ragon Institute of MGH, MIT and Harvard, Cambridge, Massachusetts, USA. \\ ${ }^{3}$ Massachusetts Institute of Technology, Department of Biological Engineering, Cambridge, Massachusetts, USA.
}

\begin{abstract}
HIV and Mycobacterium tuberculosis (M. tuberculosis) coinfection increases the risk of active tuberculosis (aTB), but how HIV infection and medications contribute to drive risk remains unknown. In this issue of the $J C l$, CorreaMacedo and Fava et al. investigated alveolar macrophages (AMs) from people living with HIV (PLWH). To mimic the earliest event in tuberculosis (TB), the authors isolated AMs from broncheoalveolar lavage (BAL) of $\mathrm{PLWH}$, healthy individuals, and healthy individuals taking antitretroviral therapy (ART) as preexposure prophylaxis (PrEP) to prevent HIV acquisition. These AMs were exposed to $M$. tuberculosis and epigenetic configuration, transcriptional responses, and cytokine production were assessed. M. tuberculosis-stimulated AMs from PLWH and from healthy individuals on PrEP showed blunted responses compared with healthy controls. While HIV infection is the major risk factor for TB, these findings suggest that ART may modulate AM responses and potentially contribute to residual risk of aTB in fully treated HIV.
\end{abstract}

Mimicking the earliest events following pathogen encounter The intersecting global pandemics of HIV and tuberculosis (TB) present a formidable public health challenge. Among people living with HIV (PLWH), TB is the leading cause of death (1). The overlap of pathology caused by these two pathogens also presents scientific challenges. Coinfection increases the risk of active TB (aTB) but the molecular determinants of this synergism are unclear. Proposed mechanisms for the higher risk of aTB include depletion of $\mathrm{CD} 4^{+} \mathrm{T}$ cells and multiple pathways of macrophage dysfunction in progressive HIV-1 infection (2). Antiretroviral therapy (ART) ameliorates but does not eliminate the enhanced risk of aTB, but the underlying mechanisms responsible for this persistent risk remain unknown. As the TB field increasingly turns to host-direct- ed therapeutics (HDTs), the mechanisms of immunologic susceptibility to aTB need to be precisely identified. Defining the impact of HIV-1 infection on potential therapeutic targets must be a high priority of active basic and translational research $(3,4)$ and a substantial portion of that effort will need to focus on the innate immune response.

In this issue of the JCI, CorreaMacedo and Fava et al. (5) interrogated the functional competence of alveolar macrophages (AMs) from PLWH. The researchers isolated AMs from broncheoalveolar lavage (BAL) and challenged them with M. tuberculosis to mimic the earliest events following pathogen encounter, a key strength of their approach. The authors showed that stimulated AMs isolated from individuals with HIV on ART have a more closed epigenetic profile as compared with

\section{Related Article: https://doi.org/10.1172/JCl148013}

Conflict of interest: The authors have declared that no conflict of interest exists.

healthy controls, which they link to blunted transcriptional responses and cytokine production. Of note, the authors also investigated phenotypes in HIV-seronegative individuals currently on preexposure prophylaxis (PrEP) to prevent HIV infection. Interestingly, they identify blunted epigenetic plasticity in the AMs from PrEP subjects, which were more similar to AMs from PLWH than to those from healthy controls. This work addresses important gaps in the understanding of TB-HIV coinfection and raises provocative questions about the potential role of ART itself.

Prior studies have suggested that there are phases to the impact of HIV infection. In the nonhuman primate model of SIV infection, an initial heightened inflammatory response in AMs following systemic SIV infection transitioned to a blunted response phenotype during chronic infection (6). This transition is thought to reflect the environment associated with HIV infection and not the direct infection of AMs, as although AMs can support HIV infection (7), it occurs in only a minority of AMs in the lungs of PLWH. Despite low amounts of infected AMs, those collected from the lungs of PLWH not on ART showed reduced induction of apoptosis after in vitro infection with M. tuberculosis that was linked to higher expression of IL-10 and suppression of the TNF- $\alpha$ response (8). Studies have sought to determine whether peripheral monocytes could model AM responses in the lung environment, with mixed results. In a comparison of the airway inflammatory profiles in PLWH not on ART and HIVseronegative individuals, despite elevated inflammatory cytokines in the peripheral blood of PLWH, there were no differences in the BAL cytokine profiles (9). However, work on peripheral monocyte-derived macrophages offers critical insights into the heterogeneity of cellular responses to $M$. tuberculosis and the central role of the differentiation environment (10). This 
research opens possibilities for more accurately modeling the $\mathrm{AM}$ responses. The work presented by Correa-Macedo and Fava et al. (5) supplies important data on the phenotype of cells at the first point of contact. Their experimental approach provides data to inform future studies with more accessible cell populations including those from the periphery, establishing a benchmark of the tissue specific response profiles to validate other model systems.

\section{Considerations and future studies}

One additional dimension of CorreaMacedo and Fava et al. (5) was the inclusion of individuals on PrEP for prevention of HIV infection. This control allowed the comparison of individuals exposed to ART both with and without concurrent HIV infection. The suggestion that ART itself may impact innate immune function is not without precedent. Previous studies have shown that some antiretroviral drugs can enhance activation of p90RSK, a kinase regulated by reactive oxygen species (11). Activation of this pathway was also observed in monocytes in a cohort of people living with HIV, most of whom were taking ART. In separate work analyzing lymphocytes, antiretroviral exposure was linked to altered immunometabolic profiles (12). Importantly, in Correa-Macedo and Fava et al. (5), all PrEP participants received tenofovir disproxil and emtricitabine, whereas there was a heterogeneous mix of agents in those participants with HIV on ART. While the overall phenotype was consistent between the two groups, it is unclear whether all ART agents would have the same impact.

A limitation of the study was the inability to assess the role of HIV itself, as all participants with HIV had viral suppression. Prior work suggests that exposure to HIV single-stranded RNA can change histone acetylation at the TNF- $\alpha$ promoter in AM cell lines (13). In contrast, studies of peripheral blood monocytes from people with chronic HIV infection identified a closed TNF- $\alpha$ promoter during high viral load, with a rise in histone 4 acetylation following HIV viral suppression that associated with increased capacity for TNF- $\alpha$ production (14). These findings from prior studies, although discordant, are consistent with the studies referenced above, where context is critical to interpretation. The importance of considering timing during the course of HIV infection, and also whether the cells are directly HIVinfected, exposed to HIV-derived innate stimuli, or if the effects are mediated by the metabolic and cytokine milieu conditioned by active HIV viremia. Population level differences, for example in the frequency of smoking or illicit drug use, may also contribute to environmental influences. Correa-Macedo and Fava and their colleagues (5) assessed environmental factors given the imbalance of smokers across their groups and did not see a marked shift in the results. Environmental factors should continue to be considered as key variables in future studies.

Future studies should interrogate how changes conditioned by ART may lead to changes in M. tuberculosis growth dynamics through impacts on nutrient utilization or other key determinants of $M$. tuberculosis survival. For example, M. tuberculosis circumvents nutrient limitation via endogenous production of tryptophan and then exploits the concurrent immunotolerant environment $(15,16)$. While it is unlikely that antiretroviral drugs directly impact $M$. tuberculosis replication, the potential for multilayer disruptions in host cell functions may have unexpected consequences for the host-pathogen interaction. Recent studies have also highlighted interactions between antiretroviral treatment, endogenous retroviruses and the immune response (17-19). It is unknown whether similar immunological dynamics driven by endogenous retroelements may have contributed to the observations made by Correa-Macedo and Fava et al. (5).

\section{Clinical implications}

There are at least two ways to consider the clinical implications of the study (5) presented by Correa-Macedo and Fava and their colleagues: (a) as a cautionary point on the use of PrEP and (b) as a cautionary point in the interpretation of epigenetic data and its correlation with clinical outcomes. In the current clinical literature, there is no clear evidence to suggest an increased risk of diagnosis with aTB among individuals using PrEP. However, it is not clear that this possibility has been investigated. The large-scale PrEP trials in sub-Saharan African women in a region of high TB endemicity do not reveal differences in TB incidence rates between the active drug and placebo arms. However, interpretation of these data is limited by the low adherence to PrEP by study participants $(20,21)$. In settings where aTB diagnosis remains rare, it will be harder to assess whether PrEP leads to an increase in risk of latent $\mathrm{TB}$ infection reactivation or acquisition. The epidemiological data definitively point to the importance of preventing HIV infection among those at risk for TB infection. HIV infection remains the strongest individual risk factor for development of aTB (2). Given the high efficacy of PrEP when used in patients with the appropriate risk profile, a theoretical risk of impaired response to TB exposure would not shift the weight of evidence away from favoring PrEP for individuals at risk.

The second possibility, highlighting the challenge of interpreting epigenetic data with clinical outcome correlates, also requires some careful thought. There is an absence of any defined clinical association between use of ART for PrEP and an increase in incidence of respiratory infections. It is possible that the epigenetics and transcriptional blunting observed have a negligible clinical consequence, or that clinical effects only manifest when there is an interaction with an unmeasured impact of HIV infection itself. Notably, in the setting of uncontrolled HIV-1 with concurrent aTB, initiation of ART is associated with a risk of immune reconstitution inflammatory syndrome (IRIS) in as many as $15 \%$ of individuals with known TB at the time of starting ART (22). These data suggest that the immune suppressive impact of viremia is much more substantial than the impact of ART, but leaves unanswered the question of residual risk of aTB in fully treated HIV, when the impacts described in CorreaMacedo and Fava et al. (5) may have a greater role in susceptibility. Given the data that antiretroviral treatments may affect host metabolism and epigenetics, future studies will need to be considered within this context, interrogating whether changes are the result of infection, medication, or the intersection of the two factors.

Address correspondence to: Eileen P. Scully, 855 N. Wolfe Street, Baltimore, Maryland 21205, USA. Phone: 410.583.2652; Email: escully1@jhmi.edu. Or to: Bryan 
D. Bryson, 25 Ames Street, Cambridge, Massachusetts 02142, USA. Phone: 617.258.7641; Email: bryand@mit.edu.

1. UNAIDS. Tuberculosis and HIV. https://www. unaids.org/en/resources/infographics/ tuberculosis-and-hiv. Updated March 24, 2020. Accessed September 21, 2021.

2. Bell LCK, Noursadeghi M. Pathogenesis of HIV-1 and Mycobacterium tuberculosis co-infection. Nat Rev Microbiol. 2018;16(2):80-90.

3. Wallis RS, Hafner R. Advancing host-directed therapy for tuberculosis. Nat Rev Immunol. 2015;15(4):255-263.

4. Zumla A, et al. Towards host-directed therapies for tuberculosis. Nat Rev Drug Discov. 2015;14(8):511-512.

5. Correa-Macedo W, et al. Alveolar macrophages from persons living with HIV show impaired epigenetic response to Mycobacterium tuberculosis. J Clin Invest. 2021;131(22):e148013.

6. Hunegnaw R, et al. Alveolar macrophage dysfunction and increased PD-1 expression during chronic SIV infection of rhesus macaques. Front Immunol. 2019;10:1537.

7. Schiff AE, et al. T cell-tropic HIV efficiently infects alveolar macrophages through contact with infected CD4+ T cells. Sci Rep.
2021;11(1):3890.

8. Patel NR, et al. Impaired M. tuberculosismediated apoptosis in alveolar macrophages from HIV+ persons: potential role of IL-10 and BCL-3. JLeukoc Biol. 2009;86(1):53-60.

9. Bunjun R, et al. Dysregulation of the immune environment in the airways during HIV infection. Front Immunol. 2021;12:707355.

10. Bryson BD, et al. Heterogeneous GM-CSF signaling in macrophages is associated with control of Mycobacterium tuberculosis. Nat Commun. 2019;10(1):2329.

11. Singh MV, et al. Senescent phenotype induced by p90RSK-NRF2 signaling sensitizes monocytes and macrophages to oxidative stress in HIV-positive individuals. Circulation. 2019;139(9):1199-1216.

12. Korencak M, et al. Effect of HIV infection and antiretroviral therapy on immune cellular functions. JCI Insight. 2019;4(12):e126675.

13. Han X, et al. Epigenetic regulation of tumor necrosis factor $\alpha(\mathrm{TNF} \alpha)$ release in human macrophages by HIV-1 single-stranded RNA (ssRNA) is dependent on TLR8 signaling. J Biol Chem. 2012;287(17):13778-13786.

14. Scully EP, et al. Innate immune reconstitution with suppression of HIV-1. JCI Insight. 2016;1(3):e85433.

15. Gautam US, et al. In vivo inhibition of trypto- phan catabolism reorganizes the tuberculoma and augments immune-mediated control of Mycobacterium tuberculosis. Proc Natl Acad Sci US A. 2018;115(1):E62-E71.

16. Zhang YJ, et al. Tryptophan biosynthesis protects mycobacteria from CD4 T-cell-mediated killing. Cell. 2013;155(6):1296-1308.

17. Banuelos-Sanchez G, et al. Synthesis and characterization of specific reverse transcriptase inhibitors for mammalian LINE-1 Retrotransposons. Cell Chem Biol. 2019;26(8):1095-1109.

18. Chuong EB, et al. Regulatory evolution of innate immunity through co-option of endogenous retroviruses. Science. 2016;351(6277):1083-1087.

19. Lima-Junior DS, et al. Endogenous retroviruses promote homeostatic and inflammatory responses to the microbiota. Cell. 2021;184(14):3794-3811.

20. Marrazzo JM, et al. Tenofovir-based preexposure prophylaxis for HIV infection among African women. N Engl J Med. 2015;372(6):509-518.

21. Van Damme L, et al. Preexposure prophylaxis for $\mathrm{HIV}$ infection among African women. $\mathrm{N} \mathrm{EnglJ}$ Med. 2012;367(5):411-422.

22. Muller M, et al. Immune reconstitution inflammatory syndrome in patients starting antiretroviral therapy for HIV infection: a systematic review and meta-analysis. Lancet Infect Dis. 2010;10(4):251-261. 